\title{
Chorioamnionitis and Neonatal Sepsis Due to Extended Spectrum Beta-Lactamase producing Escherichia Coli Infection: A Case Report
}

Saheed Shittu ${ }^{1}$, Sufia Athar ${ }^{1}$, Adila Shaukat ${ }^{1}$, and Lolwa Alansari ${ }^{1}$

${ }^{1}$ Hamad Medical Corporation

September 25, 2021

\begin{abstract}
Chorioamnionitis, a common complication of pregnancy is associated with significant maternal, perinatal, and long-term adverse outcomes. This case of placental infection leading to preterm delivery, severe neonatal sepsis, maternal wound infection, postnatal readmission, and prolonged hospital stay was caused by multi-drug resistant Extended Spectrum Beta Lactamase (ESBL)-producing Escherichia Coli
\end{abstract}

Chorioamnionitis and Neonatal Sepsis Due to Extended Spectrum Beta-Lactamase producing Escherichia Coli Infection: A Case Report

Authors:

Saheed Shittu ${ }^{1}$

Sufia Athar ${ }^{1}$

Adila Shaukat ${ }^{2}$

Lolwa Alansari ${ }^{1}$

\section{Affiliations:}

1- Obstetrics and Gynaecology Department, Al Wakra Hospital

2-Infectious Disease Unit, Division of Medicine. Al Wakra Hospital

\section{Corresponding author:}

Shittu Akinola Saheed

Department of Obstetrics and Gynaecology,

Al Wakra Hospital, Hamad Medical Corporation.

Doha, Qatar

P. O. Box 82228

Tel: +97431355516

E-mail: SShittu@hamad.qa or s2akinola@yahoo.com

\section{Abstract}


Chorioamnionitis is an acute inflammation of the membranes and chorion of the placenta typically due to ascending polymicrobial infection in the setting of membrane rupture. It is a common complication of pregnancy associated with significant maternal, perinatal, and long-term adverse outcomes. We present a case of placental infection leading to preterm delivery, severe neonatal sepsis, maternal wound infection, postnatal readmission, and prolonged hospital stay. This virulent infection was caused by multi-drug resistant Extended Spectrum Beta Lactamase (ESBL)-producing Escherichia Coli (E. Coli), which represent a major world-wide threat according to the Centre for Disease Control and Prevention (CDC). It was managed with appropriate antibiotic therapy, patient-centred approach and multidisciplinary team involvement that led to favourable maternal and neonatal outcome.

Keywords: Chorioamnionitis, wound infection, neonatal sepsis, extended spectrum beta-lactamase; E. Coli

\section{Key Clinical Message:}

ESBL-producing Enterobacteriaceae pose challenging infection control issues and are a serious global threat that requires prompt and sustained action. Screening strategies designed to monitor them could be useful in women from endemic areas to prevent perinatal transmission and the introduction of multi-resistant strains to the maternity ward.

Introduction: Chorioamnionitis is a common infection of pregnancy, typically occurring in the setting of prolonged membrane rupture or labor. It complicates $1-4 \%$ of all births in the US ${ }^{1}$. However, its frequency varies significantly with gestational age, specific risk factors and diagnostic criteria. It may be diagnosed clinically based on signs such as maternal fever, microbiologically based on amniotic fluid culture obtained by amniocentesis, or by histopathologic examination of the placenta and umbilical cord $^{2-3}$. Associated risk factors include obesity, anaemia, diabetes, prolonged duration of membrane rupture, prolonged labour, nulliparity, African American ethnicity, multiple vaginal examinations, meconium-stained liquor, smoking and drug abuse, epidural anaesthesia, compromised immunity and colonization with group B streptococcus, bacterial vaginosis, sexually transmissible genital infection and vaginal colonization with ureaplasma ${ }^{2}$.

The main preventative strategy is administration of antibiotics to women with preterm prelabour rupture of membranes which reduces the incidence of clinical chorioamnionitis, prolongs the time to delivery and improves neonatal outcomes. Optimal management of clinical chorioamnionitis includes antibiotic therapy and delivery of the infected products of conception ${ }^{5}$.

We present a case of clinical chorioamnionitis with associated fetal compromise, neonatal sepsis and severe maternal wound infection caused by multi-drug resistant extended spectrum beta-lactamase (ESBL)producing Escherichia Coli (E. Coli) and the challenges associated with its management.

Case Report: A 33-year-old Indian lady, G3P1 reported to the emergency department at 33 weeks with history of passing clear fluid vaginally for one hour. She denied having any history of fever, urinary symptoms, or vaginal discharge. Her previous delivery was by Caesarean Section (CS) in 2014. She was on metformin for gestational diabetes diagnosed at 28 weeks.

On examination she was clinically stable (Pulse-76/min, regular, BP-100/60 mm Hg, oral temperature$36.4 \mathrm{C}$ ). Systemic examination was unremarkable. Abdominal examination revealed fundal height of 32 $\mathrm{cm}$, with normal uterine tone and regular fetal heart rate. Speculum examination revealed clear leaking. Routine labs (complete blood count, C-reactive protein (CRP) and blood for type and screen) were sent. Antenatal steroids were offered, blood sugar monitoring with diet control was advised and sliding dose of insulin Injection was initiated. Oral Erythromycin was started for GBS prophylaxis. Both the patient and her family were counselled in detail regarding the conservative management and risks of chorioamnionitis and prematurity.

Her admission laboratory test results were normal (Hemoglobin-11.6 g/dL, blood group O positive, WBC$9.5 \times 10^{3} / \mathrm{Ul}, \mathrm{ANC}-7 \times 10^{3} / \mathrm{Ul}$ and CRP-7 mg/L). Ultrasound scan for growth revealed small for date fetus (growth corresponding to $<7$ th centile with oligohydramnios with normal uterine artery dopplers). She 
was clinically stable in the first 48 hours. Within 72 hours of admission, she developed tachycardia (PR$122 / \mathrm{min})$ and increased WBC $\left(12.9 \times 10^{3} /\right.$ microliter $)$ and ANC $\left(10.9 \times 10^{3} / \mathrm{Ul}\right)$ but was afebrile. CTG in the evening on the same day revealed unprovoked recurrent decelerations followed by prolonged deceleration for more than 3 minutes. In view of pathological cardiotocography findings she had emergency CS. Routine prophylactic antibiotics Cephazoline and Azithromycin were administered prior to surgery. She delivered small for date baby boy, weight $1570 \mathrm{~g}$, Apgar scores $9^{1}, 10^{5}$. The liquor was meconium-stained and foul smelling. The arterial and the venous $\mathrm{pH}$ were 7.30 and 7.36 respectively. Placental tissue was sent for culture and sensitivity and broad-spectrum antibiotics (ceftriaxone and metronidazole) were started after the surgery with the diagnosis of chorioamnionitis.

However, she had persistent tachycardia with increasing CRP levels $(420 \mathrm{mg} / \mathrm{L})$. The culture result of placental tissue and high vaginal swab (HVS) confirmed profuse growth of ESBL producing E. coli. Infectious disease team was involved and IV (intravenous) Ertapenem was commenced based on sensitivity profile of results of culture. She remained clinically stable for 72 hours and WBC counts and CRP were showing a decreasing trend. She was discharged home on IV Ertapenem to be taken on outpatient basis. However, on post-operative day 6 , she was readmitted because of wound infection and was febrile (oral temperature-38.2 C). Wound swab culture grew ESBL producing E. Coli as well and antibiotic was changed to IV Meropenem. Wound care team was involved.

After instituting Meropenem, she recovered well, and the blood counts and CRP were reduced to near normal. Meropenem was continued for 7 days. She was discharged home in clinically stable condition.

The baby was born vigorous, was initially on continuous positive airway pressure. Within few hours of birth developed signs of sepsis (pulse rate- $186 / \mathrm{m}$, blood pressure- $44 / 30 \mathrm{~mm} \mathrm{Hg}$, temperature $37.9 \mathrm{C}$ and SPO2 dropped to $93 \%$ ) with respiratory distress syndrome and was intubated and required inotropes (dopamine infusion). Broad spectrum antibiotics (Amikacin and Ampicillin) were given. Blood culture revealed gram negative bacteria within 9 hours of birth, so Meropenem was added and Ampicillin was discontinued. Fetal Echocardiography showed PDA $2.2 \mathrm{~mm}$ with bidirectional shunting and lower limit of contractility, dilated IVC with evidence of pulmonary hypertension.

Baby developed high fever (39.5 C) on second day of birth, requiring paracetamol. Total parenteral nutrition (TPN) was initiated. Laboratory results were suggestive of leucopenia (WBC $4.5 \times 10^{3} / \mathrm{uL}$ ), low platelet $\operatorname{count}\left(96 \times 10^{3} / \mathrm{uL}\right)$, high $\mathrm{CRP}(56 \mathrm{mg} / \mathrm{L})$, high serum Urea $(9.7 \mathrm{mmol} / \mathrm{L})$, high serum creatinine $(76 \mathrm{umol} / \mathrm{L})$ and high total bilirubin levels(142.3 umol/L). Blood culture result confirmed growth of ESBL- producing E. Coli. Due to persistent hypotension, higher doses of Dopamine infusion were given. On day 4, hemoglobin level dropped from $19 \mathrm{~g} / \mathrm{dl}$ to $15 \mathrm{~g} / \mathrm{dl}$ so X-ray skull and ultrasound Brain was performed and intracranial bleeding was excluded. In addition to sepsis, neonatal jaundice was noted, and ABO incompatibility was diagnosed. Lumber puncture was performed which revealed leukocytosis (216/uL and raised RBCs (11/uL). Its culture showed no growth, though was treated as meningitis.

Gradually in 10 days, the baby improved clinically. TPN was continued for 12 days and then oral feeding was commenced. Neonatal Jaundiced settled without the need of phototherapy. Antibiotic (Meropenem) was given for four weeks. Baby was discharged on 28th day of admission.

\section{Discussion}

Though, it was suspected preoperatively, the diagnosis of clinical chorioamnionitis was made at surgery as the liquor was very foul smelling. The risk factors were preterm pre-labour rupture of membranes (PROM) and gestational diabetes. There was initially no maternal fever which is present in most cases. This confirmed that knowing the risk factors can add to the diagnostic accuracy, as in all situation treatment needs to be initiated based on clinical diagnosis. Hence the definition of chorioamnionitis varies according to the key diagnostic criteria which can be clinical based on the presence of typical findings like maternal fever and tachycardia and foul-smelling liquor or vaginal discharge; microbiologic based on the culture of microbes from properly collected amniotic fluid or chorioamnion histo-pathologic based on microscopic evidence of infection or inflammation on examination of placenta or chorioamnionic specimen. Histologic chorioamnionitis is a 
more common diagnosis as it includes both subclinical and clinical chorioamnionitis. Funisitis occurs when infection/inflammation extends to the umbilical $\operatorname{cord}^{1,2-4}$.

When chorioamnionitis is suspected in the absence typical clinical signs, biochemical, serum or amniotic fluid tests may be used to diagnose subclinical chorioamnionitis. Amniocentesis for amniotic fluid culture is the best method for diagnosis of subclinical chorioamnionitis in preterm gestations. Other tests that can be obtained rapidly include white cell count, c-Reactive Protein (CRP) level, leukocyte esterase level, gram stain and glucose concentration and cytokine level (IL-6) $)^{1,5-7}$. Blood culture was not done in our patient as she initially did not have fever. Ideally this should be done prior to administration of antibiotics which should be done within an hour of recognition of severe sepsis and sepsis bundle protocol should be implemented. Critical care outreach team should be contacted if indicated ${ }^{8}$.

The maternal complications of chorioamnionitis are postpartum haemorhage, endometritis, wound infection, sepsis and death. The neonatal complications include premature birth, stillbirth, neonatal sepsis, chronic lung disease and brain injury causing cerebral palsy and other neuro-developmental disabilities ${ }^{1,5-6}$.

Route of infection is ascending infection in $96 \%$ of cases. The remainder are from haematogenous spread due to maternal septiaemia, iatrogenic from procedures like amniocentensis and CVS. Infection from the peritoneum via the fallopian tubes have been postulated ${ }^{1,2}$.

The most common organisms identified in pregnant women dying from sepsis are genital mycoplasmas, Lancefield group A beta haemolytic streptococcus and Escherichia Coli ${ }^{9}$. E. Coli belongs to the family of Enterobacteriaceae - a large order of different types of bacteria that commonly cause infections both in healthcare settings and in communities. To survive the effects of antibiotics, some Enterobacteriaceae produce extended-spectrum beta-lactamase (ESBL) enzyme that destroy and render ineffective commonly used antibiotics like penicillins and $1^{\text {st }}, 2^{\text {nd }}$ and $3^{\text {rd }}$ generation cephalosporins, but not cephamycin and carbapenems. This resistance means fewer antibiotic options available to treat ESBL producing bacterial infection and even more common infection caused by such organism may require more complex treatment requiring prolonged hospitalization and intravenous carbapenem antibiotics - a class of highly effective antibiotic agents commonly used for the treatment of severe or high-risk bacterial infections ${ }^{9-11}$. This class of antibiotics is usually reserved for known or suspected multidrug-resistant bacterial infections. Resistance that render these antibiotics ineffective are on the rise too. Therefore, appropriate and justified use of this important class of antibiotics is necessary to decrease the risk of emergence of resistance. Our patient's specimen from placental tissue and wound pus culture was resistant to commonly used antibiotics and was sensitive only to Meropenem, Ertapenem and Gentamicin. Resistance that destroys these antibiotics are on the rise too. The more we rely on this important class of antibiotics, the greater the risk of spreading resistance to them. This mandates the practice of sending appropriate specimens for culture before initiating antimicrobials as well as implementation of stewardship programs to regulate the appropriate use of antimicrobials to reduce emergence of resistance. As well as, robust infection control practices that help to prevent cross transmission of infection ${ }^{11}$.

ESBL- producing organisms were first found in Europe and the earliest cases were identified in the USA in $1988^{10}$. A pooled prevalence of faecal colonization of $14 \%$ globally was estimated by a systematic review ${ }^{9}$ and Villa $\mathrm{HE}^{10}$ et al in Argentina quoted $5.4 \%$ of pregnant women in their study had ESBL-producing E coli vaginal colonization revealing that colonization with resistant Enterobacteriaceae is significant in pregnancy. A higher prevalence of $22 \%$ was reported in South-Asia ${ }^{9}$ where our patient came from.

According to CDC, ESBL-producing Enterobacteriaceae pose challenging infection control issues and are a serious global threat that requires prompt and sustained action ${ }^{12}$. Appropriate use of antibiotic therapy under the guidance of infectious disease team is imperative. Prophylactic use of antibiotics during conservative management of PROM and prompt delivery after 34 weeks will help reduce the risk of infection. Screening strategies designed to monitor for ESBL-producing E. coli could be useful in women from endemic areas to prevent perinatal transmission and the introduction of multi-resistant strains to the maternity ward.

\section{Contributors' statements}


SS and SA conceived the idea and reviewed literature. SS, SA, AS and LA contributed to care of patient and her neonate and drafting of the manuscript. All authors read and approved the final version.

\section{Funding}

Funding for open access publication for this study is provided by Qatar National library

\section{Availability of data and materials}

All data containing relevant information to support the study findings are provided in the manuscript.

\section{Ethics approval and consent to participate}

Institutional ethics committee approval was obtained for this case report and written informed consent was obtained from all participants.

\section{Consent for publication}

Written informed consent was obtained from the patient/parents for their anonymized information to be published in this article. Documentation of the written consent will be provided to the journal upon request.

\section{Conflict of Interest}

The authors have no conflict of interest to disclose

\section{Abbreviations}

ANC : Absolute Neutrophil Count

CS : Caesarean section

CTG : Cardiotocography

HVS : High Vaginal Swab

WBC : White Blood Cell Count

\section{References}

1. Tita AT, Andrews WW. Diagnosis and management of clinical chorioamnionitis. Clin Perinatol. 2010;37(2):339-354. doi:10.1016/j.clp.2010.02.003

2. Gibbs RS, Duff P. Progress in pathogenesis and management of clinical intraamniotic infection. Am J Obstet Gynecol. 1991;164:1317.

3. Soper DE, Mayhall CG, Dalton HP. Risk factors for intraamniotic infection: a prospective epidemiologic study. Am J Obstet Gynecol. 1989;161:562.

4. Newton ER. Chorioamnionitis and intraamniotic infection. Clin Obstet Gynecol. 1993;36:795.

5. Higgins RD, Saade G, Polin RA, et al. Evaluation and Management of Women and Newborns With a Maternal Diagnosis of Chorioamnionitis: Summary of a Workshop. Obstet Gynecol 2016; 127:426.

6. Kacerovsky M, Musilova I, Hornychova H, et al. Bedside assessment of amniotic fluid interleukin-6 in preterm prelabor rupture of membranes. Am J Obstet Gynecol 2014; 211:385.e1.

7. Chaemsaithong P, Romero R, Korzeniewski SJ, et al. A point of care test for interleukin-6 in amniotic fluid in preterm prelabor rupture of membranes: a step toward the early treatment of acute intra-amniotic inflammation/infection. J Matern Fetal Neonatal Med 2016; 29:360.

8. Gilstrap LC 3rd, Cox SM. Acute chorioamnionitis. Obstet Gynecol Clin North Am. 1989 Jun;16(2):373-9. PMID: 2674804. 
9. Kennard A, Michael R. Foley and Jordan P. A Case of Hematogenous Spread of E. coli causing Clinical Chorioamnionitis Clin Med Rev Case Rep 2015, 2:017 ISSN: 2378-3656 Volume 2 | Issue 2. DOI: 10.23937/2378-3656/1410017

10.Villar HE, Aubert V, Baserni MN, Jugo MB. Maternal carriage of extended-spectrum betalactamase-producing Escherichia coli isolates in Argentina. J Chemother. 2013 Dec;25(6):324-7. doi: 10.1179/1973947813Y.0000000081. Epub 2013 Dec 6. PMID: 24091027.

11. Dolma K, Summerlin TL, Wongprasert H, Lal CV, Philips Iii JB, Winter L. Early-Onset Neonatal Sepsis with Extended Spectrum Beta-Lactamase Producing Escherichia Coli in Infants Born to South and SouthEast Asian Immigrants: A Case Series. AJP Rep. 2018 Oct;8(4):e277-e279. doi: 10.1055/s-0038-1675336. Epub 2018 Oct 29. PMID: 30377552; PMCID: PMC6205855.

12. Alrowaily N, D'Souza R, Dong S, et al. Determining the optimal antibiotic regimen for chorioamnionitis: A systematic review and meta-analysis. Acta Obstet Gynecol Scand 2021; 100:818. 